\title{
Research of a Multifunctional Fitness Equipment Monitor Based on Photoelectric Sensors
}

\author{
http://dx.doi.org/10.3991/ijoe.v12i02.5045 \\ FU Qiang \\ Northwest A\&F University, Shaanxi, China
}

\begin{abstract}
Health is an eternal topic of human concern, especially in modern times, and fitness equipment is an important part fitness routines. With the rapid development of information technology and electronic technology, using photoelectric methods to detect the physiology index of the human body has become an unescapable trend. Therefore, bringing in some advanced technology in other fields to design a portable, noninvasive, low-cost multifunctional human physiological health index monitor has great significance. This thesis first introduces the situation of fitness equipment, then studies people's demand for intelligent fitness equipment, and specifically proposes the measurement scheme of a human physical health index based on a photoelectric sensor. By studying the production principle of photoelectric volume pulse waves, this thesis sets up the experimental platform to prove the effectiveness of the proposed method. Finally, this research shows that intelligent fitness equipment like a multifunctional fitness monitor based on a photoelectric sensor can effectively improve people's exercise style and has large market application prospects.
\end{abstract}

Index Terms-Fitness Equipment, Monitor, Photoelectric Sensor

\section{INTRODUCTION}

\section{A. research background and significance}

Since the "National Fitness Plan (2011-2015)" urged people to "strengthen physical exercise," especially since Beijing's successful hosting of the Olympic Games, China's sports fitness industry has made great achievements and has stimulated enthusiasm for fitness, so fitness sports have become an indispensable part of most Chinese people's daily lives. People's demand for fitness equipment is also growsing. However, with the widespread increase of fitness groups, traditional equipment has exposed many problems with its use. Therefore, while the national need for fitness equipment is expanding, the traditional domestic equipment market urgently requires upgrading to better meet the rapid development of the demands of the fitness crowd.

Above all, to overcome the disadvantages of traditional equipment, the research and application design of intelligent fitness equipment can meet the changeable fitness and health promotion needs, can change the pattern of the fitness equipment industry that has been at a low level for a long time, and also can promote the healthy development of the fitness industry in our country, which has a great significance.

\section{B. present situation and the developing trend of fitness equipment}

Since the reform and opening-up policy, the rapid economic development and technological progress to promote the rise of China's domestic equipment industry, China's accession to the WTO, and the successful hosting of the Olympic Games in Beijing, the domestic equipment industry has brought unprecedented opportunities. However, domestic health product research and development as well as the ability for technical innovation are insufficient. Compared with Europe, the United States, and other developed countries, the gap is larger and the overall development level is lower. Through decades of development at home and abroad, great changes have taken place in fitness equipment, and traditional equipment has gradually moved in an intelligent direction.

\section{Fitness equipment}

Traditional fitness equipment is mainly composed of simple mechanical components, and its function is relatively simple. Individual fitness equipment is independent of each other and, generally, only used to exercise. However, there are a wide variety of types that can be divided into two kinds: oxygen training equipment and aerobic training equipment, according to different exercise purposes to meet the needs of different groups of people. With the enhancement of public health awareness, people propose requirements concerning the safety, entertainment, scientific focus, and other aspects in the exercise process. With traditional fitness equipment, it is more and more difficult to meet the fitness needs of modern people, so domestic and overseas entities will begin to explore more intelligent fitness equipment.

According to the survey, the intelligent fitness equipment is no longer a simple combination of the mechanical module. In the process of physical exercise, it can collect the physiological parameters of the human body and the movement parameters and provide a variety of functions, such as virtual fitness instruction, multimedia videos and so on, to meet the needs. Future intelligent fitness equipment will include electronic technology, mechanical technology, computer technology, automation technology, biomedical engineering and other multi-disciplinary fusions of sports equipment, which will become the development trend of fitness equipment. Multi-functional fitness monitors based on photoelectric sensors can satisfy people's demand for health very well. 


\section{The Principle Of MeAsuring The Human PHYSIOLOGICAL INDEX BASED ON THE OPTICAL CAPACITANCE Pulse Wave}

\section{A. Detection principle of the photocapacitance pulse wave}

According to the Lambert-Beer law and light scattering theory, with a certain wavelength beam of a substance, the absorbance and substance concentrations show a good linear relationship with optical paths, so when a constant wavelength of light irradiates on the human body, the measured transmission or reflection of the light intensity through the human tissue absorption, reflection attenuation, reflects the structural characteristics of the irradiated tissues to a certain extent. A photoelectric tracing method is a noninvasive detection method based on this principle by means of a photoelectric measure to detect blood volume changes in living tissues [1-3].

The photoelectric sensor is composed of a luminous diode (photoelectric emitter) and a photosensitive transistor (photoelectric receiver) [4], which is divided into two kinds: transmission-type and reflection-type, as shown in Figure 1.

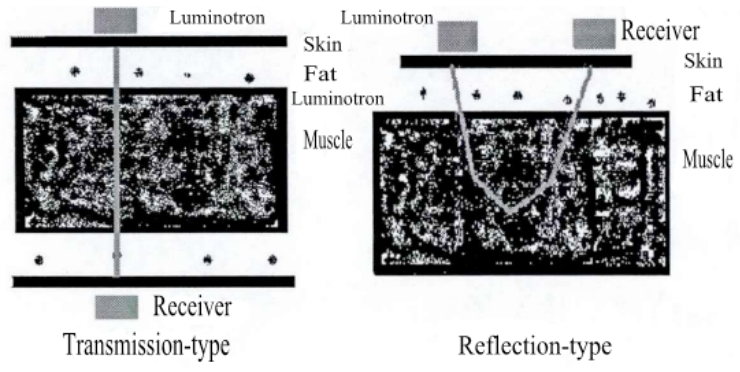

Figure 1. Sketch map of transmission and reflection measurement

The transmission-type of signal intensity is influenced by the thickness of the measurement point and the corresponding position of a luminous diode and a phototriode. The reflection-type signal intensity is influenced by the angle and distance between the luminous diode and the phototriode and the subcutaneous fat thickness of the measurement point. The transmission-type signal is stronger than the reflection-type signal about one order of magnitudes with the same incident of light intensity [5].

\section{B. the calculation principle of human physiological index based on the photoelectric pulse wave}

The basic process of life is the body's cells intake of oxygen and emission of energy produced by carbon dioxide. Blood oxygen saturation means the oxyhemoglobin percentage of the total blood hemoglobin in the blood, namely

$$
\mathrm{SaO}_{2}=\frac{\mathrm{HbO}_{2}}{\mathrm{HbO}_{2}+\mathrm{Hb}} \times 100 \%
$$

$\mathrm{SaO}_{2}$ is the blood oxygen saturation, $\mathrm{HbO}_{2}$ is the oxyhemoglobin, $\mathrm{Hb}$ is the reduced hemoglobin.

China began to study equipment using photoelectric technology to monitor human physiological parameters in 2000. So far, we have made breakthroughs in different aspects, especially accurately measuring the human body's blood oxygen saturation.
According to the theory of tissue optics, this thesis chose two wave lengths of $660 \mathrm{~nm}$ and $940 \mathrm{~nm}$ of nearinfrared light as the medium. The fingertip blood oxygen data were obtained by means of transmission, and realtime, continuous monitoring of the blood oxygen was realized. A light emitter (light emitting diode) was arranged on the side of the probe of the noninvasive pulse oxygen saturation. The incident light became emitted light through the human tissue, was received by the light detector on the other side of the probe, and then transformed the optical signal into an electrical signal so the optical absorption rate of two wavelengths could be obtained, that is

$$
R=\frac{I_{A C} 660 / I_{D C} 660}{I_{A C} 940 / I_{D C} 940}
$$

In the formula, $I_{A C}$ represents the pulsating light absorption, namely the AC component. ${ }^{I D C}$ represents the non-pulsating light absorption, namely the DC component.

The Lambert-Beer law and a large number of studies have suggested that $\mathrm{R}$ and blood oxygen content show a negative linear correlation; the lower $\mathrm{R}$ is, the greater the blood oxygen content will be. The standard relationship curve between $\mathrm{R}$ and $\mathrm{SaO}_{2}$ is shown in Figure 2, so the corresponding blood oxygen saturation can be obtained according to the standard relation curve.

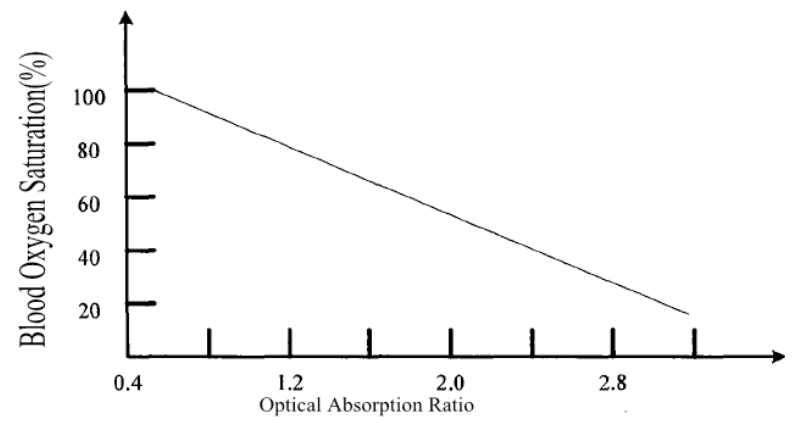

Figure 2. The standard curve between $\mathrm{R}$ and $\mathrm{SaO}_{2}$

\section{The Design Of The Multifunctional Fitness Monitor Based On PHOTOELECTRIC SENSOR}

In this paper, the measurement of a human fingertip volume pulse wave was measured by an optical capacitance pulse wave polysomnography. Optical capacitance polysomnography is a noninvasive detection method that can detect the change of blood volume in vivo by means of a photoelectric method. Pinching the photoelectric blood volume pulse wave sensor on the fingertip can monitor for a long time. Compared with the regular monitoring equipment, this sensor is more convenient and more suitable for long-term health monitoring and for dynamic monitoring because of the low cost, simple operation, avoidance of trauma, stable performance, and many other advantages.

A volume pulse blood stream exists in the peripheral vascular arterioles, capillaries and micro vein. Blood shows pulsation changes in the heart's pulsation. The waveform, amplitude and form of human body volume pulse wave contains important physiological information that reflects the status of the heart and blood vessels. This 
project used photoelectric detection technology to continuously detect the human fingertip pulse signal combined with the introduced detection principle to realize the multiple physiological index of human body measurement.

This thesis used an optical sensor, which integrated the light emitting diode and current frequency converter on one chip. The output is square, of which the duty cycle was $50 \%$, which has a good linear relationship with the light intensity received by the photodiode. The digital output can be directly connected to the microprocessor or other logic circuits. This sensor has a wide scope of work, from $-45^{\circ} \mathrm{C}$ to $80^{\circ} \mathrm{C}$. The design of this sensor adopted the method of transmission measurement. The measuring part is a human finger section, and the light-emitting diodes are $660 \mathrm{~nm}$ and $940 \mathrm{~nm}$ red light, infrared light emitting diodes. The diodes' DC power supply can cause fever and thermal expansion of the part blood vessels. Also, the ambient light, dark current, $50 \mathrm{~Hz}$ of power frequency, electromagnetic field produced by peripheral instruments, such as a high frequency electric knife, movement, and breathing, will influence the collected signal, leading to measurement error.

To eliminate the interference of noise, reduce errors and improve the accuracy of the measurement, we should make sure the insertion depth and the direction of the fingers is accurate as far as possible to reduce the pseudo differences caused by the movement of the measured individuals. We should guarantee the cleanliness of the photoelectric detector, preventing man-made inaccurate measurements. For signals gained from the photoelectric sensor first go through the low pass filter, removing the high frequency interference and then using effective methods to remove low frequency interference, including baseline drift caused by movement, breathing, and other factors to get the ideal photoelectric volume pulse wave signal.

\section{THE EXPERIMENT AND DATA ANALYSIS}

\section{A. the experimental data analysis of blood oxygen con- tent}

To determine calculation of individual fingertip blood oxygen saturation based on the principles mentioned above, first we needed to solve the light absorption ratio $\mathrm{R}$ of two wavelengths. Because the sensor chosen in this project is an optical frequency sensor and the output frequency and receiving light intensity have a good linear relationship, as shown in Table I.

$$
f_{O}=f_{D}+\operatorname{Re} \times E_{e}
$$

$f_{O}$ is output frequency, $f_{D}$ is the output frequency when light intensity is zero, usually taking $f_{D}=0$, responding to the equipment, generally taking
$\mathrm{R}_{\mathrm{e}}=2.3 \mathrm{kHz} /\left(\mu W / \mathrm{cm}^{2}\right)$. Then the formula changes to

$$
R=\frac{f_{A C} 660 / f_{D C} 660}{f_{A C} 940 / f_{D C} 940}
$$

$\mathrm{SaO}_{2}$ has a linear relation with $\mathrm{R}$, as shown in formula (4.3). We can use this formula to calculate the value of blood saturation.

$$
\mathrm{SaO}_{2}=100.6-1.3 \times R
$$

For 30 individuals, 20 men and 10 women, aged 20 to 70 , we collected photoelectric volume pulse wave data for 5 minutes, choosing 10 ideal waveform cycles using the above formula calculations as shown in Table I (see next page). We can see from Table I, oxygen saturation of the individual fingertip artery basically maintains a $98 \%$ to $99 \%$ range. The range is not large, which verifies the oxygen consumption of a fingertip artery is low and the blood oxygen saturation change is small. Compared with traditional detection methods, long-time monitoring is not harmful to the human body and its cost is much lower. Therefore, this sensor has very good application prospects and far-reaching significance.

\section{CONCLUSION}

This thesis puts forward intelligent fitness equipment, and proposes a design scheme for a multi-functional fitness monitor based on a photoelectric sensor, which changes the traditional mode of fitness and provides realtime feedback of physiological indexes. But in consideration of the complexity of fully accomplishing an intelligent fitness system and the growing demand for intelligent fitness equipment, some procedures still need to be further completed and improved.

\section{REFERENCES}

[1] Aman Chang (2014). The application of photoelectric sensor in automatic control (16).

[2] Cleary, T. (2014). Performance of dual photoelectric/ionization smoke alarms in full-scale fire tests. Fire Technology, 50(3), 753773. http://dx.doi.org/10.1007/s10694-010-0147-z

[3] Dou Shusheng. (2015). The principle and application of photoelectric sensor (4).

[4] He Tianzhu, \&amp; Lan Yu. (2013). The design of the speed meter based on photoelectric sensor (), information technology (3), 57-59.DOI:10.3969/j.issn.1009-2552.2013.03.015.

[5] Han Jisheng (2015). The design of infrared pulse detector based on single chip microcomputer (5).

\section{AUTHOR}

FU, Qiang is with Northwest A\&F University, Shaanxi, CO712100, China.

Submitted 17 September 2015. Published as resubmitted by the author 23 January 2016. 
PAPER

RESEARCH OF Multifunctional Fitness EquiPMENT Monitor BASEd ON PHOTOELECTRIC SENSOR

TABLE I.

OXYGEN CONTENT OF 10 CYCLE WAVEFORM FOR 30 INDIVIDUALS (\%)

\begin{tabular}{|c|c|c|c|c|c|c|c|c|c|c|c|c|}
\hline & $\begin{array}{c}\mathrm{SaO} 2 \\
(1)\end{array}$ & $\begin{array}{c}\mathrm{SaO} 2 \\
(2)\end{array}$ & $\begin{array}{c}\mathrm{SaO} 2 \\
(3)\end{array}$ & $\begin{array}{c}\mathrm{SaO}_{2} \\
(4)\end{array}$ & $\begin{array}{c}\mathrm{SaO} 2 \\
(5)\end{array}$ & $\begin{array}{c}\mathrm{SaO} 2 \\
(6)\end{array}$ & $\begin{array}{c}\mathrm{SaO} 2 \\
(7)\end{array}$ & $\begin{array}{c}\mathrm{SaO} 2 \\
(8)\end{array}$ & $\begin{array}{c}\mathrm{SaO} 2 \\
(9)\end{array}$ & $\begin{array}{r}\mathrm{SaO} 2 \\
(10)\end{array}$ & Av & $\mathrm{SD}$ \\
\hline 1 & 98.5 & 98.7 & 98.5 & 98.6 & 98.6 & 98.6 & 98.7 & 98.5 & 98.6 & 98.7 & 98.7 & 0.09 \\
\hline 2 & 98.5 & 98.3 & 98.6 & 98.3 & 98.6 & 98.8 & 98.5 & 98.1 & 98.3 & 99.1 & 98.5 & 0.3 \\
\hline 3 & 99.8 & 98.2 & 98.6 & 98.7 & 98.2 & 98.8 & 98.6 & 98.7 & 98.9 & 98.0 & 98.5 & 0.5 \\
\hline 4 & 98.5 & 98.7 & 98.7 & 98.6 & 98.4 & 98.5 & 98.6 & 98.6 & 98.7 & 98.5 & 98.6 & 0.09 \\
\hline 5 & 98.6 & 98.7 & 98.6 & 98.6 & 98.6 & 98.8 & 98.6 & 98.8 & 98.6 & 98.7 & 98.6 & 0.06 \\
\hline 6 & 98.6 & 98.5 & 98.5 & 98.5 & 98.7 & 98.5 & 98.6 & 98.4 & 98.4 & 98.4 & 98.8 & 0.09 \\
\hline 7 & 98.5 & 98.4 & 98.5 & 98.6 & 98.6 & 98.5 & 98.5 & 98.6 & 98.6 & 98.5 & 98.7 & 0.05 \\
\hline 8 & 98.5 & 98.3 & 98.5 & 98.5 & 98.7 & 99.3 & 98.5 & 98.7 & 98.4 & 98.5 & 98.6 & 0.26 \\
\hline 9 & 98.8 & 98.6 & 98.6 & 98.6 & 98.7 & 98.6 & 98.5 & 98.5 & 98.5 & 98.6 & 98.6 & 0.08 \\
\hline 10 & 98.8 & 98.8 & 97.1 & 98.4 & 98.6 & 98.7 & 98.9 & 98.3 & 98.4 & 98.6 & 98.6 & 0.5 \\
\hline 11 & 98.5 & 98.4 & 98.8 & 98.6 & 98.5 & 98.77 & 98.7 & 98.6 & 98.9 & 98.8 & 98.7 & 0.17 \\
\hline 12 & 98.6 & 98.3 & 98.6 & 98.9 & 98.5 & 98.1 & 98.3 & 99.1 & 98.9 & 98.7 & 98.6 & 0.30 \\
\hline 13 & 98.5 & 98.6 & 98.8 & 98.8 & 98.4 & 98.6 & 98.7 & 98.8 & 98.3 & 98.4 & 98.6 & 0.17 \\
\hline 14 & 98.3 & 98.7 & 98.4 & 98.3 & 98.5 & 98.6 & 98.6 & 98.6 & 98.1 & 98.6 & 98.5 & 0.19 \\
\hline 15 & 99.3 & 97.0 & 98.6 & 98.3 & 95.6 & 98.7 & 98.5 & 97.1 & 98.4 & 98.7 & 98.0 & 1.1 \\
\hline 16 & 98.3 & 98.6 & 99.1 & 98.4 & 98.9 & 98.6 & 98.3 & 98.1 & 98.3 & 98.8 & 98.6 & 0.3 \\
\hline 17 & 98.7 & 98.6 & 98.6 & 98.5 & 98.4 & 98.6 & 98.4 & 98.3 & 98.3 & 97.8 & 98.4 & 0.25 \\
\hline 18 & 98.6 & 98.4 & 98.6 & 98.5 & 98.6 & 97.4 & 98.6 & 98.2 & 98.4 & 98.4 & 98.7 & 0.6 \\
\hline 19 & 98.9 & 98.6 & 98.5 & 98.7 & 98.4 & 98.7 & 98.7 & 98.5 & 98.8 & 98.2 & 98.6 & 0.18 \\
\hline 20 & 98.5 & 98.7 & 98.6 & 98.5 & 98.0 & 98.5 & 98.9 & 99.0 & 98.8 & 98.6 & 98.6 & 0.28 \\
\hline 21 & 99.0 & 98.7 & 98.8 & 98.4 & 98.7 & 98.8 & 99.5 & 98.7 & 98.7 & 98.7 & 98.8 & 0.26 \\
\hline 22 & 98.7 & 98.6 & 98.6 & 98.6 & 98.6 & 98.5 & 99.5 & 98.6 & 98.4 & 98.5 & 98.7 & 0.29 \\
\hline 23 & 98.5 & 98.4 & 98.5 & 98.5 & 98.7 & 98.4 & 98.7 & 98.4 & 98.5 & 98.5 & 98.5 & 0.11 \\
\hline 24 & 98.5 & 98.7 & 98.5 & 98.3 & 98.5 & 98.7 & 98.5 & 98.5 & 98.6 & 98.5 & 98.5 & 0.1 \\
\hline 25 & 98.5 & 98.9 & 98.9 & 98.8 & 98.4 & 98.5 & 98.5 & 98.7 & 98.7 & 98.8 & 98.7 & 0.18 \\
\hline 26 & 98.8 & 98.6 & 98.0 & 98.8 & 98.5 & 98.7 & 98.8 & 98.8 & 98.6 & 98.5 & 98.6 & 0.23 \\
\hline 27 & 98.6 & 98.4 & 98.6 & 98.7 & 98.6 & 98.7 & 98.6 & 98.9 & 98.4 & 98.5 & 98.6 & 0.15 \\
\hline 28 & 98.9 & 99.1 & 97. & 98.6 & 95.9 & 99.4 & 99.5 & 98.8 & 99.0 & 99.2 & 98.6 & 1.06 \\
\hline 29 & 97.9 & 96.0 & 98.1 & 98.4 & 98.3 & 98.5 & 98.4 & 98.3 & 98.5 & 98.5 & 98.1 & 0.75 \\
\hline 30 & 98.5 & 98.6 & 98.5 & 99.1 & 95.3 & 97.4 & 98.2 & 97.9 & 98.1 & 99.2 & 98.1 & 1.12 \\
\hline
\end{tabular}

